\title{
Infrared Emission-Line Signatures of Tori in the Nuclei of Seyferts
}

\author{
Thaisa Storchi-Bergmann ${ }^{1}$ and Cláudia Winge ${ }^{1}$ \\ Instituto de Fisica, UFRGS, Campus do Vale, C.P. 15051, Porto \\ Alegre, RS, Brazil
}

Martin J. Ward ${ }^{1}$

Dept. of Physics and Astronomy, University of Leicester, University Road, Leicester LE1 7RH, UK

\author{
Andrew S. Wilson ${ }^{1}$ \\ Astronomy Program, University of Maryland, College Park, MD 20742, \\ USA
}

\begin{abstract}
We present infrared (IR) long-slit spectroscopy of two galaxies with Seyfert 2 nuclei, NGC 2110 and the Circinus galaxy, both known from previous studies to exhibit anisotropic high-excitation gaseous emission. In the 'unified models' for Seyferts, this anisotropy is due to collimation of the ionizing nuclear radiation by an optically thick molecular torus which surrounds the nucleus. We have looked for signatures of this torus and its collimating effect in the IR ( $J$ and $K$ bands), taking advantage of the reduced relative attenuation at these wavelengths. The two galaxies are found to show extended emission in the lines [Fe II] $\lambda 1.257 \mu \mathrm{m}, \mathrm{Pa} \beta$, $\mathrm{H}_{2} \lambda 2.12 \mu \mathrm{m}$, and $\mathrm{Br} \gamma$, both along the collimation axis as well as perpendicular to it. The [Fe II] line is found to trace the high-excitation emission, and, in the case of NGC 2110 , is more extended along the collimation axis. The $\mathrm{H}_{2}$ emission, although consistent with the presence of a molecular torus, extends for hundreds of parsecs and the derived kinematics is consistent with circular rotation in the plane of the galaxies. The emission-line ratios $[\mathrm{Fe} \mathrm{II}] / \mathrm{Pa} \beta$ and $\mathrm{H}_{2} / \mathrm{Br} \gamma$ are much smaller in Circinus (respectively $\sim 0.5$ and 1$)$ than in NGC $2110(\sim 7$ and $\geq 4$, respectively), and indicate the presence of a nuclear starburst in the former galaxy. IR rotation curves were determined for both galaxies. In the case of NGC 2110, the IR rotation curve shows different behavior than the rotation curve determined from optical lines, which shows the importance of obscuration effects in the study of the gas kinematics.
\end{abstract}

\footnotetext{
${ }^{1}$ Visiting Astronomer, Cerro Tololo Inter-American Observatory. CTIO is operated by AURA, Inc., under cooperative agreement with the National Science Foundation.
} 


\section{Introduction}

The popular 'unified-model' scenario for Seyfert galaxies (see Antonucci 1993 for a recent review) invokes an optically thick molecular torus which surrounds the nuclear engine. This torus both collimates the ionizing radiation and obscures the nucleus from direct view in Seyfert 2 galaxies. This is an appealing model, but there is so far little direct evidence for the existence of the optically thick material itself. Detection of the molecular hydrogen line of $\mathrm{H}_{2} v=1-0, \mathrm{~S}(1)$ (rest wavelength $\lambda_{0}=2.122 \mu \mathrm{m}$ ) should reveal the presence of hot molecular gas within the obscuring torus. If an $\mathrm{H}_{2}$-emitting disk-like structure could be detected elongated perpendicular to the gaseous cone (observed in a number of Seyfert $2 \mathrm{~s}$ ) or radio axis, this would provide very strong support for the unified model. Narrow-band imaging studies by Blietz et al. (1994) have indeed shown that in NGC 1068 the line emission is spatially extended almost perpendicular to the cone. Similar results were found for NGC 4945 (Moorwood et al. 1996) and in the Circinus galaxy (Maiolino 1997, these proceedings).

With the goal of looking for spectral signatures of the torus and its collimating effect in the IR, we obtained long-slit spectra in the $J, H$, and $K$ bands of a number of Seyferts with anisotropic high-excitation emission, presumably due to ionization and excitation by nuclear radiation collimated by the torus. In this work, we present the results for NGC 2110 and Circinus, the two galaxies for which the emission was detected reaching the largest angular distances from the nuclei.

NGC 2110 is an early-type Seyfert 2 showing high-excitation gas extending up to 10 ' from the nucleus, with an ' $\mathrm{S}$ '-shaped morphology, approximately coincident, close to the nucleus, with a jet-like radio feature at position angle $P A=10^{\circ}$ (Ulvestad \& Wilson 1983, Mulchaey et al. 1994). Wilson \& Baldwin (1985), using optical long-slit spectroscopy, have found extended ionized gas in normal rotation about the minor axis, but with the kinematic center displaced by $\sim 1^{\prime \prime} .7$ south of the optical continuum nucleus. Throughout this paper, we will adopt a distance to NGC 2110 of $31.2 \mathrm{Mpc}$ (using the systemic velocity from Wilson \& Baldwin 1985, for $H_{0}=75 \mathrm{~km} \mathrm{~s}^{-1} \mathrm{Mpc}^{-1}$ ), corresponding to a scale of $151 \mathrm{pc} \operatorname{arcsec}^{-1}$.

The Circinus galaxy is a nearby spiral (Sb-d) with a nuclear spectrum characteristic of both starburst and Seyfert activity (Oliva et al. 1994), and shows the closest ionization cone, with a circumnuclear starburst ring (Marconi et al. 1994). It is a strong $\mathrm{H}_{2} \mathrm{O}$ megamaser, and shows radio lobes orthogonal to the Galactic plane, at $P A \approx 114^{\circ}$ (Elmoutie et al. 1995). We adopt a distance to Circinus of $4 \mathrm{Mpc}$ (Marconi et al. 1994), corresponding to a scale of $19 \mathrm{pc} \operatorname{arcsec}^{-1}$.

\section{Observations}

Long-slit spectra in the $J$ and $K$ bands were obtained using the Infrared Spectrograph (IRS) on the 4-m telescope of the Cerro Tololo Interamerican Observatory on 1995 November 1 and 2 and on 1996 March 2. The slit width projects to $1^{\prime \prime}$ on the sky, and was oriented in two position angles, one along the radio axis and the other perpendicular to it. The useful slit length in the spatial direc- 


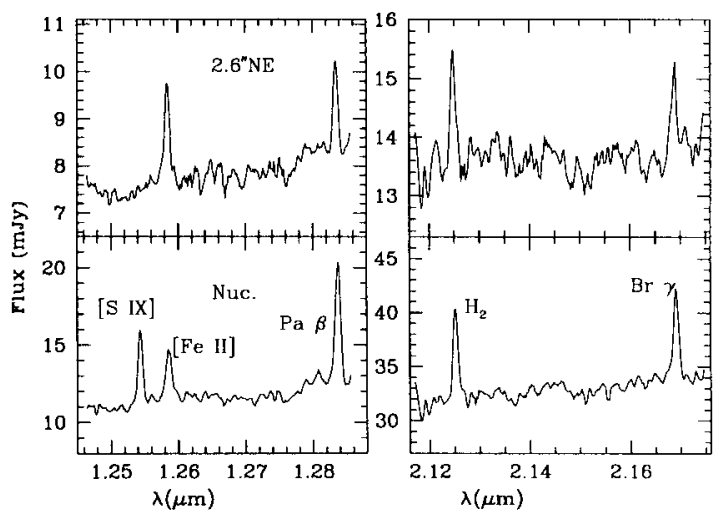

Figure 1. Two extracted spectra of Circinus $\left(1^{\prime \prime} \times 0^{\prime \prime} .7\right)$ at $J$ (left) and $K$ (right) bands, from the nucleus and from $2^{\prime \prime} .6 \mathrm{NE}$ along $P A=24^{\circ}$.

tion was $15^{\prime \prime}$. Two gratings were used, yielding resolution $\Delta v \approx 500 \mathrm{~km} \mathrm{~s}^{-1}$ and $\Delta v \approx 150 \mathrm{~km} \mathrm{~s}^{-1}$, respectively.

The spectra were flux calibrated using standard stars and divided by normalized atmospheric standard stars. Spectra were then extracted, binning together two pixels, corresponding to $0^{\prime \prime} .7$ on the sky. Figure 1 shows a sample of the extracted spectra of Circinus.

\section{Results}

NGC 2110: Emission is found extending up to $\sim 600 \mathrm{pc}$ from the nucleus. The full width at half maximum (FWHM) of [FeII], after correction for the instrumental profile, is $\sim 500 \mathrm{~km} \mathrm{~s}^{-1}$, while for $\mathrm{H}_{2}$, the FWHM is $\leq 200 \mathrm{~km} \mathrm{~s}^{-1}$. The emission-line ratio $[\mathrm{Fe} I \mathrm{II}] / \mathrm{Pa} \beta$ reaches very high values at the nucleus $(\sim 7)$ as compared with similar data obtained for NGC 1068, for example $(\sim 1$; Ward et al. 1987). The origin of the [Fe II] emission has been assumed to be gas exposed to nuclear X-ray emission or interaction with a nuclear outflow or jet. This last hypothesis is consistent with the observed broadening of the profile.

Figure 2 shows the rotation curve obtained from the IR lines. It is interesting to compare this rotation curve with the optical one obtained by Wilson \& Baldwin (1985): we do not confirm, in the IR, the displacement of $1^{\prime \prime} .7$ found by these authors between the optical and kinematic nucleus. Our rotation curve is approximately symmetric relative to the optical nucleus within $0^{\prime \prime} .5$. This result indicates that the displacement found by Wilson \& Baldwin is due to a larger obscuration in the optical and does not support a different location for the kinematic and optical nucleus.

Circinus: The emission in most lines extends beyond the inner $15^{\prime \prime}$ covered by the slit length, corresponding to $\sim 100 \mathrm{pc}$ to each side of the nucleus. Only the coronal line of [S IX] at $1.252 \mu \mathrm{m}$ is unresolved (at a limiting spatial resolution of $\sim 20 \mathrm{pc}$ ), as previously found by Oliva et al. (1994). All emission lines are 


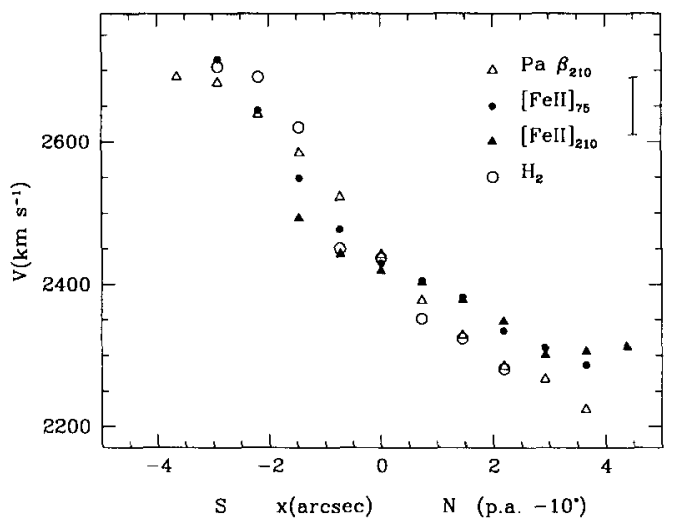

Figure 2. IR rotation curve for NGC 2110

narrow, with $\mathrm{FWHM} \leq 170 \mathrm{kms}^{-1}$. The ratio $[\mathrm{Fe} \mathrm{II}] / \mathrm{Pa} \beta$ is smaller than in NGC 2110 and is consistent with the presence of a starburst at the nucleus. It increases perpendicularly to the plane, which can be understood as [FeII] emission tracing the high-excitation gas along the radio axis.

The rotation curve of Circinus, obtained from the central wavelengths of the IR emission lines shows a turnover radius located at only $\sim 80 \mathrm{pc}$ from the kinematic nucleus (which is displaced relative to the optical nucleus by $\sim 20 \mathrm{pc}$ to the SW). This indicates a large central mass concentration, and a calculation using a simple spherical mass model gives $2 \times 10^{8} M_{\odot}$ for the mass inside $80 \mathrm{pc}$.

A more detailed analysis of the data discussed here will be presented in a forthcoming paper.

\section{References}

Antonucci, R. R.J. 1993, ARAA, 31, 473.

Blietz, M., Cameron, M., Drapatz, S., Genzel, R., Krabbe, A., van der Werf, P., Sternberg, A., \& Ward, M. 1994, ApJ, 421, 92.

Elmoutie, M., Haynes, R.F., Jones, K.L., Ehle, M., Beck, R., \& Wielebinski, R. 1995, MNRAS, 275, L53.

Marconi, A., Moorwood, A.F., Origlia, L., \& Oliva, E. 1994, ESO Messenger, No. $78,20$.

Moorwood, A.F.M., \& Oliva, E. 1994, ApJ, 429, 602.

Mulchaey, J.S., Wilson, A.S., Bower, G.A., Heckman, T.M., Krolik, J. H., \& Miley, G. K. 1994, ApJ, 433, 625.

Oliva, E., Salvati, M., Moorwood, A. F. M., \& Marconi, A. 1994, A\&A, 288, 457.

Ulvestad, J.S., \& Wilson, A.S. 1983, ApJ, 264, L7.

Ward, M.J., Geballe, T., Smith, M., Wade, R., \& Williams, P. 1987, ApJ, 316, 138.

Wilson, A.S., \& Baldwin, J. A. 1985, ApJ, 289, 124. 